\title{
FATORES ASSOCIADOS À VULNERABILIDADE DA NÃO ADESÃO DO DISTANCIAMENTO SOCIAL DE TRABALHADORES NA COVID-19
}

\section{FACTORS ASSOCIATED WITH THE VULNERABILITY OF NON-ADHERENCE TO THE SOCIAL DISTANCING OF WORKERS IN COVID-19}

\author{
Francisco Mayron Morais Soares ${ }^{1}$ * Kirley Kethellen Batista Mesquita ${ }^{2}$ * Camilo Hugo Freitas \\ de Andrade A $^{3}$ Dayllana Stefanny Lopes Lima Feitosa ${ }^{4}$ Tatyane Oliveira Rebouças ${ }^{5 *}$ Patrícia \\ Giselle Freitas Marques $^{6}$ Ana Carolina de Melo Farias Teixeira ${ }^{6}$
}

\section{RESUMO}

Objetivo: refletir sobre os fatores associado as vulnerabilidades de diferentes domínios como individual, social e programática de trabalhadores informais e os impactos da não adesão do distanciamento social nesse cenário de pandemia. Método: Estudo Reflexivo overview, que contou com o auxílio de publicações recentes relacionadas a esta proposta, sobretudo de pesquisas realizadas no Brasil e em outros países. Resultados: O estudo permitiu tecer reflexões sobre o impacto da pandemia nos trabalhadores informais associados vulnerabilidades e seus domínios Considerações finais: Esta reflexão pode contribuir para repensar a saúde e segurança e fomentar reflexões críticas acerca da desigualdade social no enfrentamento da pandemia.

Palavras-chave: Trabalhadores Pobres; Enfermagem; Análise de Vulnerabilidade; Área Programática de Saúde; Infecções por Coronavirus.

\begin{abstract}
Objective: to reflect on the factors associated with the vulnerabilities of different domains such as individual, social, and programmatic of informal workers and the impacts of non-adherence to social distancing in this pandemic scenario. Method: Reflective Study Overview, which had the help of recent publications related to this proposal, especially research conducted in Brazil and other countries. Results: The study allowed reflections on the impact of the pandemic on informal workers associated with vulnerabilities and their domains Final considerations: This reflection can contribute to rethink health and safety and foster critical reflections on social inequality in tackling the pandemic.
\end{abstract}

Keywords: Poor Workers; Nursing; Vulnerability Analysis; Health Programmatic Area; Coronavirus Infections.

\footnotetext{
${ }^{1}$ Enfermeiro. Mestre em Enfermagem. Docente da Graduação em Enfermagem da Faculdade UNINTA Itapipoca. Itapipoca, Ceará, Brasil. ${ }^{2}$ Enfermeira. Mestranda em Enfermagem pela Universidade Federal do Ceará -UFC. Fortaleza, Ceará, Brasil.

${ }^{3}$ Graduando em Enfermagem da Faculdade UNINTA Itapipoca. Bolsistas de Iniciação Científica. Itapipoca, Ceará, Brasil.

${ }^{4}$ Enfermeira. Mestre em Enfermagem. Aquiraz, Ceará, Brasil.

${ }^{5}$ Enfermeira. Mestre em Ensino em Saúde. Centro de Hematologia e Hemoterapia do Ceará. Fortaleza, Ceará, Brasil.

${ }^{6}$ Enfermeira. Fortaleza, Ceará, Brasil.
} 


\section{INTRODUÇÃO}

Em março de 2020, a Organização

Mundial de Saúde classificou o Coronavírus

Disease 2019 (COVID-19) como uma pandemia. Para tanto, a população e os serviços de saúde adotaram mudanças rigorosas devido à rápida transmissibilidade do vírus, pelo alto número de pacientes críticos e a complexidade dos cuidados neles empregados, configurando-se com um problema de saúde pública ${ }^{(1,2)}$.

A COVID-19 surgiu no mês de dezembro de 2019 na região da China e amostras colhidas no trato respiratório, identificaram a presença do vírus ${ }^{(3,4)}$. Pela rápida disseminação nos países $\mathrm{e}$ as constantes mudanças sociais e epidemiológicas que impactaram diretamente nas condições clínicas de pacientes acometidos pela COVID-19, ela apresentou-se como um grande desafio para o mundo e os serviços de saúde, pois diversos países apresentaram altos índices de doentes, o que demandou de forma urgente medidas preventivas como manobra estratégica de controle $^{(5,6,7)}$.

Em junho de 2020, no mundo, foram confirmados aproximadamente 6,5 milhões de casos da COVID-19 e quase 400 mil mortes, tais números torna-se alarmante devido ao alto teor de transmissibilidade ${ }^{(8)}$. Nesse sentindo, em consonância com as orientações sugeridas pela OMS, os países têm instituídos medidas preventivas como estratégia de diminuição e provável estabilização de casos.

Devido ao aumento rápido do grau epidêmico, as relações interpessoais, tornaram-se um grande vetor na disseminação do vírus, mediante a tal suposição, foram sugeridas medidas simples como higienização das mãos (com água e sabão ou álcool a 70\%), uso de máscaras, proteger nariz e boca ao espirrar ou tossir (etiqueta respiratória), além do distanciamento social. Tais medidas foram utilizadas e teve como foco a redução da transmissão do vírus e tentar evitar o colapso dos sistemas de saúde, entretanto estas medidas causaram crises sociais devido à vulnerabilidade individual, social e programática, principalmente nos trabalhadores informais que dependem das relações interpessoais para manutenção da renda mensal. $(9,10,11)$.

Não obstante, a população, em especial os trabalhadores informais desenvolveram muitos sentimentos e, estes, são vistos de forma negativa, como medo, angústia, crises de ansiedade, impotência de 
trabalhadores informais (principalmente para os provedores do lar), angústia, preocupações e em especial para manutenção de provisão do lar, problemas alimentares, conflitos internos, sociais e familiares e, por fim, mas não menos importante os problemas mentais ${ }^{(12)}$. Diante do exposto, tais sentimentos são derivados das medidas preventivas ocasionados pelo distanciamento e isolamento sociais impostos por meio do governo, que mesmo sendo estratégico para contenção do vírus, impactaram na geração de conflitos pessoais e familiares, que por vezes, devido as condições financeiras e sociais supracitadas, não conseguiram seguir ao distanciamento $\mathrm{e}$ isolamento social, e continuaram a trabalhar de forma ilegal. ${ }^{(4,12,13)}$

Diante do exposto, este estudo teve como objetivo refletir sobre os fatores associado as vulnerabilidades de diferentes domínios como individual, social e programática de trabalhadores informais e os impactos da não adesão do distanciamento social nesse cenário de pandemia.

\section{MÉTODOS}

Estudo reflexivo, do tipo overview, para o qual se buscou realizar uma reflexão sobre a promoção da saúde e reunir os conhecimentos sobre o tópico em estudo a fim de construir a síntese reflexiva ${ }^{(14)}$.

As reflexões foram tecidas à luz da Teoria Social da Individualização ${ }^{15}$, que parte da premissa da socialização em uma sociedade de risco.

Devido à incipiência da temática, optou-se por incluir artigos e manuais que dispusessem da mesma. A busca ocorreu nas bases de dados: Base de dados de Enfermagem (BDENF), National Library of Medicine and National Institutes of Health (PUBMED/Medline), Cumulative Index of Nursing and Allied Health Literature (CINAHL), Web of Science, Excerpta Medica dataBASE (EMBASE), Scientific Electronic Library Online (SciELO) e Cochrane por meio do seguinte cruzamento: "Trabalhadores Pobres" ans "Enfermagem" and "Análise de Vulnerabilidade" and "Área Programática de Saúde” and Infecções por Coronavirus".

Foram estabelecidos os critérios de elegibilidade para a pesquisa, considerandose os seguintes tipos de estudos: 1) artigos sobre a temática covid-19; 2) que abordassem a vulnerabilidade individual, social e programática no enfrentamento da covid-19, sem restrição temporal e de idioma. 
A busca procedeu-se pelas seguintes etapas, inicialmente foram realizados as leituras dos títulos dos artigos que contemplavam os critérios supracitados, posteriormente procedeu-se com a leitura dos resumos dos artigos e posteriormente ocorreu a leitura dos artigos na integra para, de fato, incluir no estudo, trabalhos que contemplassem o objeto de estudo.

As reflexões propostas foram embasadas à luz (principalmente) da literatura nacional atinente ao tema. Tal afirmativa versa sobre as condições instituídas no Brasil. A apresentação das explanações e reflexões a serem tecidas se deu na forma de eixos condutores sobre o tema, advindos de interpretações da literatura e impressões reflexivas dos autores. Estas interpretações foram dirigidas pela compreensão do tema no âmbito nacional, ou seja, o "estado da arte". Ademais, como não houve nenhuma interação de pesquisa direta/aplicada, excluiu-se a necessidade de submeter o estudo a trâmites éticos.

Conforme preconizados pela Resolução 510/2016 do Conselho Nacional de Saúde que envolve pesquisados com dados de domínio público, procedeu-se a condução do estudo.

\section{RESULTADOS}

\section{Análise reflexiva}

O estudo permitiu tecer reflexões sobre as vulnerabilidades e seus dominios frente ao enfrentamento da pandemia, baseados à luz da Teoria Social da Individualização. Tal cenário impactou diretamente o adesão do distaciamento social, e por ser multifatorial, surge como uma atenção necessário no que cerne a temática. Para tanto, foram divididas duas categorias reflexivas.

\section{Vulnerabilidade individual e social na pandemia de enfrentamento covid-19}

Diante da atua situação que se encontra o Brasil, que vive mais uma epidemia, a desigualdade social representa abismos ainda mais evidentes de forma nacional e global que, de forma estratificada, têm-se a apresentação da população pobre com maiores índices de impacto e vulnerabilidades ${ }^{(16)}$.

Por conta da limitação de recursos de prevenção individual e coletiva, sanitárias e, sobremodo, econômicas, acabam ocasionando dificuldades na prática de distanciamento social e contribuem para a disseminação do vírus e o aumento da taxa 
de internação hospitalar, pois até mesmo serviços básicos de saúde têm limitado o acesso da população ${ }^{(4,17)}$.

Diante do atual panorama supracitado, gera-se uma reflexão crítica frente as dificuldades e injustiças enfrentadas pela população, e gera um debate que enfatiza o contexto das vulnerabilidades tanto social como programática ${ }^{(16)}$.

Para aproximar a temática, serão tecidos os conceitos e definições acerca da Vulnerabilidade e seus domínios (individual, social e programática (11). Entende-se vulnerabilidade, como a condição comprometida de indivíduos ou grupos específico acerca da sua compreensão, com isso, podem apresentar dificuldades para sumarizar seus interesses pessoais, problemas cognitivos, diminuição do nível educacional por deficiências de recursos etc. $(16,17,18)$.

Em relação aos domínios, a vulnerabilidade individual compreende os componentes biopsiosocioemocionais nas relações sociais, a vulnerabilidade social concerne nos aspectos culturais e oportunidade de aquisição de bens e trabalhos, e a vulnerabilidade programática está, de fato, associada a recursos sociais necessários, efetivos e democráticos, para que as pessoas vivam em condições de prevenir doenças ou suas complicações

O comprometimento da condição de vulnerabilidade é composto pela combinação dos domínios e conforme o sofrimento expresso, bem como a forma da condução em relação a lidar com as facilidades e dificuldades vivenciadas ${ }^{(11,16)}$.

A dimensão individual, traz à tona, o trabalhador informal e o enfrentamento social em relação à forma de empreender e a relação social de vendas, pois o desemprego estrutural, contribuiu para o aumento da não adesão.

Nesse contexto, é expresso uma reflexão acerca dos mais diversos cenários que estes trabalhadores enfrentam no decorrer da sua construção econômica para a sobrevivência e sua adaptação na continuidade da geração de sua fonte de renda.

De formas, as medidas de proteção individual e coletiva forma implementadas, mas, devido a disparidade econômica, os mais pobres, em especial os trabalhadores informais, sofreram impactos que ainda não conseguem ser mensurados. Portanto, recomenda-se estudos para avaliação de tal fenômeno.

Ademais, as condições sociais 
empregadas as populações mais pobres refletem na não adesão do distanciamento social.

\section{Desigualdade social e o impacto do trabalho em relação a vulnerabilidade programática}

Para garantir a sustentabilidade econômica de profissionais, foi instituído o trabalho de home office, sobremodo, este não se aplica a população mais pobre e os trabalhadores informais, que, de forma não rara, vivem pelas ruas, como vendedores ambulantes, pequenos agricultores, e pequenos comércios. Tal aspecto de trabalho citado colide com os subempregos e os empregos informais, causando impactos a classe mais pobre ${ }^{(18)}$.

Nessa perspectiva, a desigualdade econômica sustenta a necessidade de trabalho aos trabalhadores informais e, de forma mais significativa, pela condição já programática de contas mensais, contribuíram para a continuidade das atividades laborais informais.

A vulnerabilidade programática, que compromete as condições de provisão condições mínimas para garantir a qualidade de vida, manutenção e prevenção de doenças e agravo, com associação a recursos sociais necessários, efetivos e democráticos torna-se um grande problema, uma vez que inúmeros fatores influenciam na aquisição dessa igualdade $^{(11,15,19)}$.

A pobreza extrema, as condições não favoráveis de moradia, os problemas psicológicos, as dependências químicas, sociais e emocionais ${ }^{19}$ geraram impactos que deverão no futuro, serem expostos em estudos de avaliação econômica em saúde.

Não obstante, os trabalhadores informais não têm renda fixa, e por conseguinte, como houve a diminuição da prestação de serviços, principalmente de grandes empresas, acabaram dando continuidade ao seu trabalho informal, a fim de garantir a renda, mas tal atividade se configura como não adesão do distanciamento social e provavelmente aumentou o risco de transmissibilidade do vírus.

Alguns estudos refletem sobre os fatores que influenciam a baixa ou não adesão ao distanciamento social, e tais limitações geram grande impactos em diversos pelos teóricos. A falta de emprego formal, a falta ou má alimentação, a ausência de provimentos do lar, morados de casas locadas, e até mesmo as tarifas de impostos que eram para serem abolidas nesse período 


\section{ARTIGO DE REVISÃO}

também contaram como tais fatores. A seguir será apresentado um quadro 1 que evidenciam estudos que falam sobre os fatores.

Quadro 1 - Exemplos de estudos na temática sobre a desigualdade e vulnerabilidades. Brasil, 2020

\begin{tabular}{|l|l|}
\hline \multicolumn{1}{|c|}{ Autores } & \multicolumn{1}{|c|}{ Título } \\
\hline Aldaiza De Oliveira Sposati(19) & $\begin{array}{l}\text { COVID-19 Revela a Desigualdade de Condições da } \\
\text { Vida dos Brasileiros }\end{array}$ \\
\hline $\begin{array}{l}\text { Magno Nunes Farias, Jaime Daniel } \\
\text { Leite Junior(15) }\end{array}$ & $\begin{array}{l}\text { Vulnerabilidade social e covid-19: considerações a } \\
\text { partir da terapia ocupacional social }\end{array}$ \\
\hline $\begin{array}{l}\text { Tadeu Alencar Arrais,Adriano } \\
\text { Rodrigues De Oliveira, Juheina } \\
\text { Lacerda Viana, Diego Pinheiro } \\
\text { Alencar,Tathiana Rodrigues } \\
\text { Salgado, Jorge Pires De Morais } \\
\text { Neto,Maria Ester De Souza }\end{array}$ & $\begin{array}{l}\text { Debate: Celeiros da pobreza urbana: suplementação de } \\
\text { renda e isolamento social em ambientes } \\
\text { metropolitanos nos tempos Pandêmicos. }\end{array}$ \\
\hline $\begin{array}{l}\text { Armin Mathis }{ }^{(4)} \\
\text { Angela }\end{array}$ & $\begin{array}{l}\text { Cadastro único, espelho da pobreza e vulnerabilidade } \\
\text { social no pará, e as possibilidade de seu uso como } \\
\text { Instrumento de gestão no combate Da pandemia de } \\
\text { covid-19 }\end{array}$ \\
\hline $\begin{array}{l}\text { Marcia Dahmer } \\
\text { Quinzani }{ }^{(20)}\end{array}$ & $\begin{array}{l}\text { O avanço da pobreza e da desigualdade social como } \\
\text { efeitos da crise da Covid-19 e o estado de bem-estar } \\
\text { social }\end{array}$ \\
\hline Tricia Viviane Lima Calmon ${ }^{(22)}$ & $\begin{array}{l}\text { As condições objetivas para o enfrentamento ao covid- } \\
\text { 19: abismo social Brasileiro, o racismo, e as } \\
\text { perspectivas de desenvolvimento social como } \\
\text { Determinantes }\end{array}$ \\
\hline
\end{tabular}

Os exemplos apresentados no Quadro 1 demonstram, por meio de publicações aleatória, a disseminação de informações acerca da temática.
Diante do exposto, os estudos abordam de forma geral as dificuldades $\mathrm{e}$ as necessidades para alcançar um patamar igualitário para as classes sociais. Pois a desigualdade social, racial e econômica é um 
contributo para a diminuição da adesão do distanciamento social.

As medidas de distanciamento social seguem duas direções opostas, uma no objetivo de contenção da disseminação do vírus, outra no contexto do enfraquecimento da economia global, e destaca-se os trabalhadores informais que mais sofreram nesse período, o que os deixam mais vulneráveis e aumentam significativamente as diferenças das classes sociais, principalmente em países em desenvolvimento, como o Brasil.

Não obstante, vale considerar que tal fenômeno pandêmica é temporário, embora ainda existam apenas estimativas de sua duração. Tal estudo, contribui para o melhor entendimento das vulnerabilidades e contextos enfrentados nesse papel de pandemia. E se entende que os danos são grandes, mas com o tempo, irão ocorrer processos de normalização.

É preeminente investimentos para o desenvolvimento de políticas públicas para o amparo as populações mais vulneráveis em situação de risco. E faz-se necessário a implantação de programas que auxiliem os trabalhadores na condução de seu trabalho frentes as dificuldades que de forma, não rara, apresentam-se no cotidiano.
Ainda são incipientes os estudos que avaliem os impactos econômicos nas populações vulneráveis e estes, são, importantes para efetivar e informar a atual concepção do prisma brasileiro da desigualdade social.

Vale ressaltar que empregar políticas públicas voltados a todos, mas em especial ao menos favorecidos, contribuirá para o melhor desenvolvimento de outros surtos e situações difíceis.

Reforça-se que o distanciamento social é importante, e embora, mesmo com danos mais evidentes nos trabalhadores informais, deverá ser mantida para que diminua o tempo de crise.

\section{CONSIDERAÇÕES FINAIS}

Entende-se que esse período de pandemia difícil tem causado impactos nas populações mais pobres em especial nos trabalhadores informais e que o distanciamento social muitas vezes não é cumprido pelo fato das vulnerabilidades e seus domínios, conforme refletido.

Ademais, destaca-se que os principais fatores versam principalmente na situação econômica (provedores do lar), social (mais pobres). E acredita-se que, após esse período de pandemia, deverão ser implementadas 


\section{ARTIGO DE REVISÃO}

medidas educacionais para evitar eventuais transtornos futuros.

Por fim, este estudo não tem como intenção finalizar as discussões sobre a temática, mas fomentar reflexões críticas acerca da desigualdade social no enfrentamento da pandemia.

\section{REFERÊNCIAS}

1 - World Health Organization (WHO). Health workers exposure risk assessment and management in the context of COVID19 virus. [Internet]. Geneva: WHO; 2020 [acesso em 06 jun 2020]. Disponível em: https://apps.who.int/iris/bitstream/handle/10 665/331340/WHO-2019-nCov-

HCW risk assessment- 2020.1eng.pdf? sequence $=1 \&$ is Allowed $=\mathrm{y}$

2 - Moren DM, Daszak P, Taubenberger JK. Escaping Pandora's Box-Another Novel Coronavirus. N Engl J Med [Internet]. 2020 Fev [cited 2020 abr 05]; 382:1293-1295.

Disponível em: https://doi.org/10.1056/NEJMp2002106

3 - González de Molina Ortiz F, Gordo Vidal F, Estella García A, Morrondo Valdeolmillos $\mathrm{P}$, Fernández Ortega J, Caballero López $\mathrm{J}$ et al. Recomendaciones de "no hacer" en el tratamiento de los pacientes críticos de los grupos de trabajo de la Sociedad Española de Medicina Intensiva, Crítica y Unidades Coronarias (SEMICYUC). Medicina Intensiva. 2018;42(7):425-443.

4 - Farias H. O avanço da Covid-19 e o isolamento social como estratégia para redução da vulnerabilidade. Espaço e
Economia. 2020;(17)

5 - Wang, D.; Hu, B.; Hu, C.; et al. Clinical characteristics of 138 hospitalized patients with 2019 novel coronavirus-infected pneumonia in Wuhan, China. JAMA. 2020. https://doi.org/10.1001/jama.2020.1585.

6 - Yang X, Yu Y, Xu J, Shu H, Xia J, Liu H, et al. Clinical course and outcomes of critically ill patients with SARS-CoV-2 pneumonia in Wuhan, China: a singlecentered, retrospective, observational study. Lancet Respir Med. 2020. https://doi.org/10.1016/s22132600(20)30079-5.

7 - Lin H, Liu W, Gao H, Nie J, Fan Q. Trends in Transmissibility of 2019 Novel Coronavirus-Infected Pneumonia in Wuhan and 29 Provinces in China. SSRN Electronic Journal. 2020;

8 - OPAS/OMS Brasil - Folha informativa COVID-19 (doença causada pelo novo coronavírus) | OPAS/OMS [Internet]. Pan American Health Organization / World Health Organization. 2020 [cited 4 June 2020]. Available from: https://www.paho.org/bra/index.php?option= com content $\&$ view $=$ article $\&$ id $=6101:$ covid 1 9\&Itemid $=875$

9 - Gasmi A, Noor S, Tippaitote T, Dadar M, Mensel A, Bjørklund G. Individual risk management strategy and potential therapeutic options for the COVID-19 pandemic. Clin Immunol 108409 [Preprint]. 2020 Apr. [cited Apr 22, 2020]. Avaliable from:

https://doi.org/10.1016/j.clim.2020.108409

10 - Cheng VCC, Wong S-C, Chen JHK, Yip CCY, Chuang VWM, Tsang OTY, et al. 
Escalating infection control response to the rapidly evolving epidemiology of the coronavirus disease 2019 (COVID-19) due to SARS-CoV-2 in Hong Kong. Infect Control Hosp Epidemiol [Preprint].2020 Mar.

11 - Ayres J, Calazans GJ, Saletti Filho HC, França Jr I. Risco, vulnerabilidade e práticas de prevenção e promoção da saúde. In: Campos G, Minayo MCS, Akerman M, Drumond Jr M, Carvalho YM, organizadores. Tratado de Saúde Coletiva. São Paulo: Editora Fiocruz; 2006. p. 375417.

12 - Miranda FMA, Santana L de L, Pizzolato AC, Saquis LMM. Condições de trabalho e o impacto na saúde dos profissionais de enfermagem frente a Covid19. Cogitare enferm. [Internet]. 2020 [acesso em 06 jun 2020]; 25. Disponível em: http://dx.doi.org/10.5380/ce.v25i0.72702.

13 - Silva M, Procópio I. A fragilidade do sistema de saúde brasileiro e a vulnerabilidade social diante da COVID-19 [Internet]. Periodicos.unifor.br. 2020 [cited 4 June 2020]. Available from: https://periodicos.unifor.br/RBPS/article/vie $\underline{\mathrm{w} / 10724}$

14 - Polit DF, Beck CT, Hungler BPH, Thorell A. Fundamentos de Pesquisa em Enfermagem. Porto Alegre: Artmed; $9^{\mathrm{a} e d .}$ 2018.

15 - Peixoto Rodrigues L, Felipe Narciso P. TEORIA SOCIAL: vinte lições fundamentais. 2020.

16 - Farias M, Leite Junior J. Vulnerabilidade social e COVID-19: considerações a partir da terapia ocupacional social. 2020.

17 - Souza E, Boigny R, Ferreira A, Alencar C, Oliveira M, Ramos Jr. A. Vulnerabilidade programática no controle da hanseníase: padrões na perspectiva de gênero no Estado da Bahia, Brasil. Cadernos de Saúde Pública. 2018;34(1).

18 - Rodrigues Natália Oliveira, Neri Anita Liberalesso. Vulnerabilidade social, individual e programática em idosos da comunidade: dados do estudo FIBRA, Campinas, SP, Brasil. Ciênc. saúde coletiva [Internet]. 2012 Aug [cited 2020 June 04] ; 17( 8 ): 2129-2139.

19 - Sposati A. COVID-19 Revela a Desigualdade de Condições da Vida dos Brasileiros. NAU Social. 2020;11(20):101.

20 - Grandi M. Colapso e determinismo escalar em tempos pandêmicos: reflexões preliminares sobre a casa, o "isolamento social" e o déficit habitacional. Revista Tamoios. 2020;16(1).

21 - Al-Alam E, Massuquetti A, Azevedo A. Os efeitos da liberalização comercial na pobreza das nações: uma análise por meio da renda real e do bem-estar. Revista Econômica. 2018;19(2).

22 - Calmon T. As condições objetivas para o enfrentamento ao COVID-19: abismo social brasileiro, o racismo, e as perspectivas de desenvolvimento social como determinantes. NAU Social. 2020;11(20):131.

Recebido: 2020-06-30

Aceito: 2020-07-30 